\title{
Assessing Blood Brain Barrier Permeability in Traumatic Brain Injury Research
}

\author{
George P. Liao, Benjamin M. Aertker, Daniel J. Kota, Karthik S. Prabhakara, Philippa \\ Smith, Robert A. Hetz, Hasen Xue, Supinder Bedi, Scott D. Olson and Charles S. Cox Jr* \\ Department of Pediatric Surgery, University of Texas Health Science Center at Houston, 6431 Fannin Street, MSB 5.230, \\ Houston, TX 77030, USA
}

*Corresponding Author: E-mail: Charles.S.Cox@uth.tmc.edu; Tel.: +1-713-500-7307; Fax: +1-713-500-7296

Received: March 06, 2015; Revised: July 09, 2015Y; Published: September 05, 2015

\begin{abstract}
The blood brain barrier plays an important role in traumatic brain injury, serving at the crossroads of secondary injury and potential therapies. In regards to trauma, this barrier contains an array of cellular and molecular components that protect the central nervous system from derangements in water homeostasis and inflammation. Preclinical and clinical assays have been developed to describe and quantify blood brain barrier permeability in relation to the integrity of these blood brain barrier components and the handling of edema. This review will discuss both preclinical and clinical molecular and imaging techniques that are used to assess blood brain barrier function and recovery following traumatic brain injury.
\end{abstract}

\section{Keywords}

Extravasation; MRI; Alexa Fluor; Evans Blue; Edema

\section{Introduction}

Blood brain barrier (BBB) permeability and cerebral edema play crucial roles in the progression of secondary injury following Traumatic Brain Injury (TBI), and are clinically associated with increased intracranial pressure and compromised cerebral perfusion [1]. The pathological consequence of BBB compromise is the exposure of the normally immunologically privileged central nervous system to an influx of neuroinflammatory cells and proinflammatory cytokines [2,3]. Neuroinflammation promotes vasogenic edema and co-exists with cytotoxic edema from cellular dysfunction and dysregulation of intracellular volume $[4,5]$.

The injury penumbra is the at-risk area adjacent to the injury site where the BBB is likely in transition between healthy and dysfunctional cerebral microvasculature, and can be evaluated in the preclinical setting via excess tissue water, BBB component protein up and downregulation and imaging the extravasation of various markers. Clinically, BBB compromise can be estimated via microdialysis catheters or external ventricular drains as ratios between brain and serum total protein or albumin levels [6]. Clinical visualization of penumbral BBB permeability in vivo has been begun to be possible using imaging modalities such as diffusion tensor magnetic resonance imaging and emerging in vivo fluorescence technologies [7]. This review will discuss BBB identification and use as a therapeutic target to protect and restore brain tissue following TBI. 


\section{Physiology}

The BBB is lined with capillary endothelial cells that lack fenestrations and are attached by tight junctions (comprised of claudin-5, occludin, zona ocludens-1 and VE-cadherin) that together restrict transport $[8,9]$. Adenosine triphosphate-binding cassette transporters prevent drug penetration across the BBB. In comparison with peripheral endothelial cells, the BBB endothelial cells are much more energy dependent, containing five to ten times the amount of mitochondria [3]. These endothelial cells are further covered by astrocyte end processes and have basal lamina shared with luminal pericytes. Astrocytes express high levels of aquaporin-4, a water channel protein that is known to be involved in the clearance of edema $[10,11]$.

The mechanical forces of the primary injury transmitted to BBB disrupts blood flow and energy supply, compromising the barrier to the central nervous system, allowing for the passage of neuroinflammatory cells, potent osmotic proteins such as albumin, and neuro excitatory molecules such as glutamate [12]. Subsequently, inflammation, edema and excitotoxicity contribute to clinically relevant secondary brain injury that may even manifest in epileptic events [13]. The resident astrocytes and microglia within the central nervous system are activated and also contribute to secondary injury, leading to further neuronal death [14].

\section{Preclinical Measurements of BBB Permeability}

A number of invasive strategies have been employed by preclinical investigators to assess the status of the BBB. The strategies include measuring brain water content, BBB components and extravasation. The first strategy is using brain water content, the simplest method of indirectly assessing the degree of BBB dysfunction and offers global differences between treatments, but is subject to variations in animal sacrifice technique and post mortem handling of brain tissues [15]. The second strategy is to measure the components involved in forming the BBB, such as perivascular astrocytes, tight junction proteins such as claudin-5, occludin and zona occludens-1, as well as the components responsible for maintaining and restoring water hemostasis, such as aquaporin-4 $[10,16,17]$. While this strategy can describe how individual components of the BBB change following injury and treatment, the effect of other components and the function of the BBB cannot be directly assessed.

The third strategy is to use imaging with various molecular probes to detect the degree of extravasation present due to BBB permeability. Evans Blue dye extravasation is a common assay used in TBI research. Evans Blue has affinity to serum albumin $(66 \mathrm{kDa})$. Following injury, the BBB, with pore size normally allowing for molecules of general 0.4 kDa mass to pass, becomes disrupted, allowing for Evans Blue to leave the vasculature and extravasate into the interstitial tissue. The amount of extravasation can be quantified by extraction $[18,19]$. Despite wide use, the Evans Blue assay is limited by the relatively large molecular size of albumin, the reliance on detection by absorbance via a wide fluorescence excitation spectrum, and harsh extraction process that destroys the brain tissue and precludes further histologic analysis on the same tissue sample.

Alexa Fluor 680 (Lifetechnologies, Carlsbad, CA) is a far-red dye that can be bioconjugated to various molecular weight dextrans as well as other molecules and has been used in investigations for drug transit across the BBB [20]. High resolution (approaching $20 \mu \mathrm{m}$ ) infrared laser scanners can detect subtle changes in signal intensities of Alexa Fluor 680 between adjacent anatomical structures in brain tissue samples. We previously demonstrated that Alexa Fluor 680 reduces the non-specific signal between sham and injury to $7 \%(p<0.001)$ when compared to Evans Blue, an eightfold reduction (Figure 1). Infrared image scanning 
precludes the need of detaching contralateral hemispheres for normalization and decreases signal to noise. Maintaining intact brain architecture allows for the use of additional fluorescent markers to evaluate cell integrity and junctional proteins comprising the BBB. The tissue can be further processed for sectioning and staining.

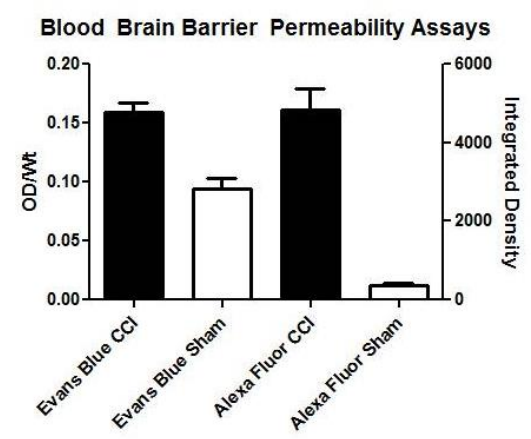

Figure 1. Non-specific signals are reduced with Alexa Fluor 680 conjugated to 10 kDA dextran compared to Evans Blue dye as evidenced by the high signal from sham animals receiving Evans Blue. Evans Blue control cortical injury $(\mathrm{CCl})(n=11)$, Evans Blue Sham $(n=6)$, Alexa Fluor $\mathrm{CCI}(n=9)$, Alexa Fluor Sham $(n=10)$. The $Y$ axis $\mathrm{OD} / \mathrm{Wt}$ refers to optical density (absorbance) per brain weight. The $\mathrm{X}$ axis Integrated Density refers to the cumulated signal detected by the far-red scanner across the brain slices.

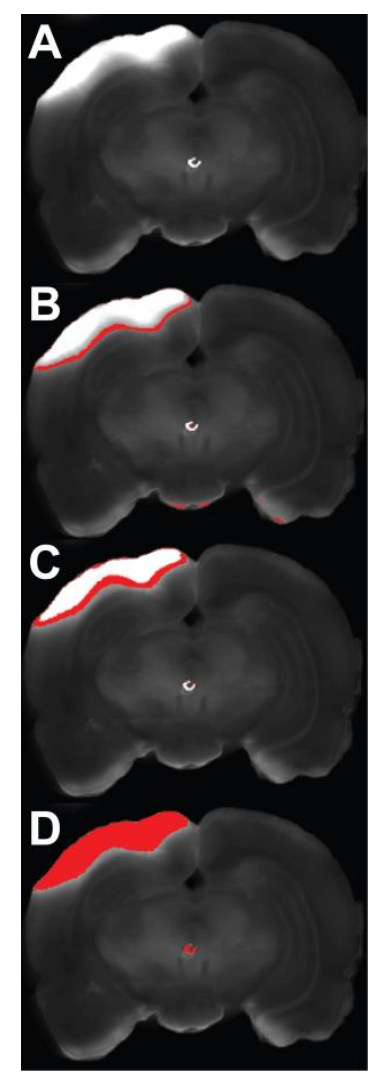

Figure 2. Four ranges of intensity thresholds applied to the same coronal brain slice for a rat using ImageJ software. White areas demonstrate tissue edema, red areas represent the Alexa Fluor 680 conjugated to 10 kDA dextran signal captured at 700nm using a LI-COR Odyssey CLx infrared laser scanner. (A) No intensity threshold applied. (B) The low + narrow intensity range of 4000-5000, represents areas of low Alexa Fluor extravasation and suggests areas with a lower degree of BBB compromise within the at-risk injury penumbra. (C) The low + less narrow intensity range of 5000-7500 represents areas of higher Alexa Fluor extravasation and suggests areas with a higher degree of BBB compromise within the at-risk injury penumbra. (D) Intensity range of 5000-Maximum representing all areas of Alexa Fluor extravasation.

We demonstrated that Alexa Fluor 680 dye conjugated to $10 \mathrm{kDa}$ dextran can be used in preclinical studies of TBI to suggest the location and degree of BBB permeability associated with at-risk penumbral 
regions. Rats undergoing controlled cortical injury were designated to vehicle versus intravenous cell therapy groups and were sacrificed at 72 hours. Prior to sacrifice the brains were perfused with Alexa Fluor 680 conjugated to $10 \mathrm{kDa}$ dextran, then following extraction were sectioned coronally into $1 \mathrm{~mm}$ slices. These slices were then imaged using a LI-COR Odyssey CLx infrared laser scanner (LI-COR, Lincoln, Nebraska) at 700 and $800 \mathrm{~nm}$. Raw images were then stacked, processed and analyzed in batch using Fiji [21], the fully open source version of ImageJ $1.48 \mathrm{p}$ (http://imagej.nih.gov/ii).

A low and narrow intensity threshold range identified a rim of Alexa Fluor signal in the area of the penumbral tissue that may be the subtle transition zone of BBB permeability [22] (Figure 2). This threshold range demonstrated significant signal differences between the treated and control TBI rats. Wider threshold ranges, with greater Alexa Fluor signal identified tissue associated with intraparenchymal contusions or microvascular hemorrhage.

Other infra-red imaging and immunohistochemical stragegies have also been used for extravasation studies. Infra-red imaging contrast agents of various sizes have also been used by preclinical investigators in direct and indirect BBB permeability assays. An example of a small agent is the P-glycoprotein efflux transporter contrast molecule, rhodamine $800(0.5 \mathrm{kDa})[23,24]$. Larger molecular weight permeability markers include IRDye $800 \mathrm{CW}$ which can vary in size from $15 \mathrm{kDa}$ and upwards, depending on conjugation [25]. Fluorescein isothiocyanate conjugated to dextran or albumin has been used, as well as probing for IgG penetration into the central nervous system after injury [26-30]. Two-photon microscopy with tetramethylrhodamine-dextran is less invasive, and has been used in rat and mouse models of stroke and neoplasia for in vivo cerebral blood flow and edema information [31]. Translating this technique to the BBB in severe TBI animal models may be challenging due to the condition of injured tissue and the required imaging window.

Some investigators have used a radiolabeling strategy to quantify movement of material across the BBB. Briefly, the isotope alpha- $\left[{ }^{14} \mathrm{C}\right]$-aminioisobutyric acid is a tracer introduced into the circulation along with a red blood cell labeling agent, technetium $-99^{m}$. The amount of tracer that has moved out of the vasculature across the BBB can be described by a transfer constant, $K \mathrm{i}$ by differentiating the parenchymal tracer concentration from the intravascular tracer, using co-localization with red blood cells labeled with technetium-99 ${ }^{m}[32,33]$.

\section{Clinical Measurement of BBB Permeability}

BBB permeability can be clinically measured through central nervous system (CNS) and peripheral fluid sampling or via imaging. BBB disruption has been defined as a total cerebrospinal fluid (CSF) protein concentration to total plasma protein concentration ratio greater than 0.007 [6,34]. Proteins such as S100B are released by astrocytes in areas of tissue injury and can be measured via jugular vein or peripherally [35]. The principles of identifying areas of BBB compromise and edema can be translated into neuroimaging. In 2006, Lescot et al. used computed tomography to measure volume, weight and specific gravity of contused and non-contused areas in patients with severe TBI [36]. Positron emission tomography (PET) can identify the injury penumbra using a metabolic strategy. Anaerobic metabolism can be identified as hypodense gray matter and also localized by measuring oxygen extraction fraction, cerebral metabolic rates of oxygen and glucose consumption [37].

Magnetic resonance imaging (MRI) has emerged as an imaging technique that can be used in both preclinical and clinical studies of BBB permeability following TBI. Dynamic contrast enhanced MRI uses extravasation of low-molecular weight contrast agents along with repeated T1-weighted imaging to 
evaluate the degree of BBB disruption and has been used in human and animal models in a variety of applications from tumors to stroke [38-41]. Investigators using MRI with diffusion weighted imaging (DWI) technology have been able to identify temporal and regional differences in edema following TBI in rabbits. The authors were able to differentiate between vasogenic and cytotoxic edema based on higher or lower apparent diffusion coefficients respectively [42]. Newcombe et al. applied diffusion tensor MRI to at-risk contusions in acute post TBI patients and found a pattern of concentric regions of varying diffusion similar to our Alexa Fluor findings. The authors suggested that the outermost rim of hypodensity may represent a 'traumatic penumbra' which may be rescued by effective therapy [43].

BBB studies involving mild TBI due to repeat concussions have been studied using a combination of serum S100, auto-antibodies to S100B and diffusion tensor MRI technology focused on white matter damage $[44,45]$. Blast shock wave models of mild TBI using animal models suggest that the primary injury not only causes damage from free radical induced oxidative stress resulting tight junction, pericytes and astrocyte end-feet disruption, but also causes vascular lesions. Both of these mechanisms may progress into long term neuroinflammatory damage and chronic traumatic encephalopathy (CTE) [46-48].

MRI imaging technology has emerged as an excellent, non-invasive tool to follow the evolution of both mild and severe TBI in both pathogenesis and potential therapy. Blood pool contrast agents such as the large gadofosveset trisodium has been used in MRI as a method to examine BBB permeability noninvasively [49]. Originally developed for vascular surgery, this particular contrast agent has strong binding to albumin, long half-life, thus much like Evans Blue can be used in humans using MRI with the advantage of repeated measures and localization of leakage [50]. This particular contrast agent has been used both pre-clinically and clinically to examine BBB breakdown in brain tumors, multiple sclerosis, utilization for traumatic brain injury is emerging [51,52]. Smaller agents have been developed, such as gadolinium diethylenetriaminepentaacetate $(0.5 \mathrm{kDa})$.

Within the MRI imaging, cellular contrast agents are now being used to show specific cellular infiltration through the BBB. Inflammatory cells entering the human brain through the BBB can be labeled with superparamagnetic iron oxide particles (SPIO) and perfluorocarbons [53]. This technology can help investigators describe the cellular neuroinflammatory response across the $B B B$, but also help track the response to potential therapies.

While MRI imaging has been the most common clinical imaging modality for BBB permeability, other techniques are emerging. Recently, fluorescence-based time resolved optical detection method for assessment of the BBB has found success in humans. This technique is based on monitoring of the fluorescence following the excitement of indocyanine green dye. Following the washout of indocyanine green dye circulating in the brain and may offer a useful adjunct or alternative to MRI in describing BBB permeability $[54,55]$.

\section{Targets and role of cellular therapy}

Strategies to protect and stabilize the BBB include the blockade of molecules or receptors to inflammatory cytokines as well as the blockade of glutamate, transforming growth factor $\beta$, fibrinogen, thrombin, vascular endothelial growth factor, bradykinin, histamine and metalloproteinases. Glucose transporter modulation has also been investigated in reducing the co-transport of water into injured tissue [56]. As TBI progresses from the primary to secondary injury, the number of deranged components of the $\mathrm{BBB}$ and CNS increases along with the number of infiltrating cells and molecules. Therefore, no single molecular target or pharmacological agent is likely to adequately rescue the BBB and protect the CNS from 
further injury.

Cellular therapy offers a treatment strategy that can potentially sense and respond to multiple signals with multiple neuroprotective and neuroregenerative responses. BBB studies utilizing Evans Blue as well as immunohistochemistry have previously demonstrated that cell based therapies can modulate the neuroinflammatory response following TBI resulting in BBB preservation [57-59]. Of note, Pati et al. demonstrated in mouse models of TBI that mesenchymal stem cells exert perivascular protective effects to the penumbral BBB via increased junctional protein (VE-cadherin and occludin) expression [60]. Walker et al showed increased localization/organization of occludins to the microvasculature [61]. The Cox lab is currently using diffusion tensor MRI imaging to evaluate the response to cell therapy in acute setting for severe TBI in pediatric and adult clinical trials (NCT01851083 and NCT 01575470 respectively).

\section{Conclusions}

The BBB continues to be an important therapeutic target in TBI research. Preclinical and clinical BBB assays continue to evolve. Brain water content and BBB component assays can now be complemented by an array of imaging techniques that quantify BBB related extravasation, function and even infiltration of inflammatory cells. Non-invasive MRI and fluorescence based optical detection show promise for translational research by providing a platform for conducting preclinical studies that can be unified with clinical outcome measures.

\section{References}

[1] J.L. Alves, J. Neurosci. Res. 92(2) (2014) 141-147.

[2] P. Barzo, A. Marmarou, P. Fatouros, K. Hayasaki, F. Corwin, J. Neurosurg. 87(6) (1997) 900-907.

[3] S.C.Thal, W. Neuhaus, Arch. Med. Res. 45(8) (2014) 698-710.

[4] A. Pasco, L. Lemaire, F. Franconi, Y. Lefur, F. Noury, J.-P. Saint-Andre, J.-P. Benoit, P.J. Cozzone, J.-J. Le Jeune, J. Neurotrauma 24(8) (2007) 1321-1330.

[5] A. Marmarou, Neurosurg. Focus 22(5) (2007) E1.

[6] M.M. Saw, J. Chamberlain, M. Barr, M.P.G. Morgan, J.R. Burnett, K.M. Ho, Neurocrit. Care 20(2) (2014) 209-216.

[7] K. Wang, B. Liu, J. Ma, J.Chin. Med. (Engl) 127(10) (2014) 1964-1968.

[8] P. Grammas, J. Martinez, B. Miller, Expert. Rev. Mol. Med. 13 (2011) e19.

[9] D. Vajtr, O. Benanda, J. Kukačka, R. Pruša, L. Houstava, P. Toupalik, R. Kizek, Physiol. Res. 58(2) (2009) 263-268.

[10] J.A. Stokum, D.B. Kurland, V. Gerzanich, J. Marc Simard, Neurochem. Res. 40(2) (2015) 317-328.

[11] K. Taya, C.R. Marmarou, K. Okuno, R. Prieto, A. Marmarou, J. Neurotrauma 27(1) (2010) 229-239.

[12] A.R. Frattalone, G.S. Ling, Neurosurg. Clin. N. Am. 24(3) (2013) 309-319.

[13] D. Lozano, G.S. Gonzales-Portillo, S. Acosta, I. de la Pena, N. Tajiri, Y. Kaneko, C.V. Borlongan, Neuropsychiatr. Dis. Treat. 11 (2015) 97-106.

[14] J. Lu, S.J. Goh, P.Y.L. Tng, Y.Y. Deng, E.-A. Ling, S. Moochhala, Front. Biosci. (Landmark Ed) 14 (2009) 3795-3813.

[15] W.W. van den Brink, A. Marmarou, C.J. Avezaat, Acta Neurochir. Suppl. (Wien) 51 (1990) 261-262.

[16] S. Khosrow Tayebati, F. Amenta, D. Tomassoni, CNS Neuro . Disord. Drug Targets ( 2015).

[17] J. Wen, S. Qian, Q. Yang, L. Deng, Y. Mo, Y. Yu, Exp. Ther. Med. 8(3) (2014) 881-886.

[18] M. Imer, B. Omay, A. Uzunkol, T. Erdem, P.A. Sabanci, A. Karasu, S.B. Albayrak, A. Sencer, K. Hepgul, M. Kaya, Neurol. Res. 31(9) (2009) 977-981. 
[19] M. Khan, Y.B. Im, A. Shunmugavel, A.G. Gilg, R.K. Dhindsa, A.K. Singh, I. Singh, J. Neuroinflammation 6:32 (2009).

[20] M. Nuriya, T. Shinotsuka, M. Yasui, Cereb. Cortex 23(9) (2013) 2118-2126.

[21] J. Schindelin, I. Arganda-Carreras, E. Frise, V. Kaynig, M. Longair, T. Pietzsch, S. Preibisch, C. Rueden, S. Saalfeld, B. Schmid, J.-Y. Tinevez, D.J. White, V. Hartenstein, K. Eliceiri, P. Tomancak, A. Cardona, Nat. Methods 9(7) (2012) 676-682.

[22] G.P. Liao, S.D. Olson, D.J. Kota, R.A. Hetz, P. Smith, S. Bedi, C.S. Cox Jr, J. Surg. Res. 190(2) (2014) 628633.

[23] N.H. On, P. Kiptoo, T.J. Siahaan, D.W. Miller, Mol. Pharm. 11(3) (2014) 974-981.

[24] N.H. On, S. Savant, M. Toews, D.W. Miller, J. Cereb. Blood Flow Metab. 33(12) (2013) 1944-1954.

[25] N.H. On, F. Chen, M. Hinton, D.W. Miller, Pharm. Res. 28(10) (2011) 2505-2515.

[26] M. Krueger, I. Bechmann, K. Immig, A. Reichenbach, W. Härtig, D. Michalski, J. Cereb. Blood Flow Metab. 35(2) (2015) 292-303.

[27] B.T. Hawkins, Y.H. Gu, Y. Izawa, G.J. del Zoppo, J. Cereb. Blood Flow Metab. 35 (2015) 985-992.

[28] F.Y. Jalal, Y. Yang, J.F. Thompson, T. Roitbak, G.A. Rosenberg, J. Cereb. Blood Flow Metab. 35 (2015) 1145-1153.

[29] J.H. Kim, D.W. Lee, B.Y. Choi, M. Sohn, S.H. Lee, H.C. Choi, H.K. Song, S.W. Suh, Brain Res. 1595 (2015) 156-165.

[30] J. Badaut, D.O. Ajao, D.W. Sorensen, A.M. Fukuda, L. Pellerin, Neuroscience 285 (2015) 215-226.

[31] S.M. Schwarzmaier, M. Gallozzi, N. Plesnila, J. Neurotrauma 32(13) (2015) 990-1000.

[32] J. Zhang, G.B. Sadowska, X. Chen, S.Y. Park, J.-E. Kim, C.A. Bodge, E. Cummings, Y.-P. Lim, O. Makeyev, W.G. Besio, J. Gaitanis, W.A. Banks, B.S. Stonestreet, FASEB J 29 (2015) 1739-1753.

[33] X. Chen, G.B. Sadowska, J. Zhang, J.-E. Kim, E.E. Cummings, C.A. Bodge, Y.-P. Lim, O. Makeyev, W.G. Besio, J. Gaitanis, S.W. Threlkeld, W.A. Banks, B.S. Stonestreet, Neurobiol. Dis. 73 (2015) 118-129.

[34] K.M. Ho, S. Honeybul, C.B. Yip, B.I. Silbert, J. Neurosurg. 121(3) (2014) 674-679.

[35] B. Arfvidsson, T.K. Nilsson, L. Norgren, Clin. Chem. Lab. Med., 53(1) (2015) 111-117.

[36] T. Lescot, V. Degos, A. Zouaoui, Crit. Care Med. 34(12) (2006) 3029-3033.

[37] H.M. Wu, S.C. Huang, P. Vespa, D.A. Hovda, M. Bergsneider, J. Neurotrauma 30(5) (2013) 352-360.

[38] A. Montagne, S.R. Barnes, M.D. Sweeney, M.R. Halliday, A.P. Sagare, Z.Zhao, A.W. Toga, R.E. Jacobs, C.Y. Liu, L. Amezcua, M.G. Harrington, H.C. Chui, M. Law, B.V. Zlokovic, Neuron 85(2) (2015) 296-302.

[39] W. Li, J.A. Long, L.T. Watts, Z. Jiang, Q. Shen, Y. Li, T.Q. Duong, PLoS One 9(12) (2014) e114173.

[40] N.J. Heyer, J.H. Derzon, L. Winges, C. Shaw, D. Mass, S.R. Snyder, P. Epner, J.H. Nichols, J.A. Gayken, D. Ernst, E.B. Liebow, Clin. Biochem. 45(13-14) (2012) 1012-1032.

[41] Z. Merali, J. Leung, D. Mikulis, F. Silver, A. Kassner , Transl. Stroke. Res. 6(1) (2015) 39-49.

[42] X.E. Wei, Y.Z. Zhang, Y.H. Li, M.H. Li, W.B. Li, J. Neurotrauma. 29(14) (2012) 2413-2420.

[43] V.F. Newcombe, G.B. Williams, J.G. Outtrim, D. Chatfield, M. Gulia Abate, T. Geeraerts, A. Manktelow, H. Room, L. Mariappen, P.J. Hutchinson, J.P. Coles, D.K. Menon, J. Cereb. Blood Flow Metab. 33(6) (2013) 855-862.

[44] N. Marchi, J.J. Bazarian, V. Puvenna, M. Janigro, C. Ghosh, J. Zhong, T. Zhu, E. Blackman, D. Stewart, J. Ellis, R. Butler, D. Janigro, PLoS One 8(3) (2013) e56805.

[45] E. Bargerstock, V. Puvenna, P. Iffland, T. Falcone, M. Hossain, S. Vetter, S. Man, L. Dickstein, N. Marchi, C. Ghosh, J. Carvalho-Tavares, D. Janigro, PLoS One 9(7) (2014) e101477.

[46] J.E. Bailes, A.L. Petraglia, B.I. Omalu, E. Nauman, T. Talavage, J. Neurosurg. 119(5) (2013) 1235-1245.

[47] A.K. Shetty, V. Mishra, M. Kodali, B. Hattiangady, Front. Cell Neurosci., 8 (2014) 232.

[48] H. Ling, J. Hardy, H. Zetterberg, Mol. Cell Neurosci. 66(Pt B) (2015) 114-122.

[49] F.L. Giesel, A. Mehndiratta, M. Essig, Eur. Radiol. 20(10) (2010) 2461-2474.

[50] M. Essig, K. Nikolaou, J.F. Meaney, Eur. Radiol. 17 Suppl 2 (2007) B30-37. 
[51] J. Puig, G. Blasco, M. Essig, J. Daunis-i-Estadella, G. Laguillo, A.M. Quiles, S. Remollo, K. Bergmann, C. Joly, L. Bernado, J. Sánchez-González, S. Pedraza, Eur. Radiol. 23(4) (2013) 1093-1101.

[52] E. Fisher, D.S. Reich, Neurology 81(3) (2013) 202-203.

[53] G. Weise, G. Stoll, Front. Neurol. 3 (2012) 178.

[54] A. Liebert, et al., Conf. Proc. IEEE Eng. Med. Biol. Soc. 2013 (2013) 3040-3042.

[55] N. Horie, Y. Fukuda, T. Izumo, K. Hayashi, K. Suyama, I. Nagata, Acta Neurochir. (Wien) 156(5) (2014) 919-926.

[56] D. Shlosberg, M. Benifla, D. Kaufer, A. Friedman, Nat. Rev. Neurol. 6(7) (2010) 393-403.

[57] P. A. Walker, S.K. Shah, F. Jimenez, K.R. Aroom, M.T. Harting, C.S. Cox Jr., Surgery 152(5) (2012) 790793.

[58] P.A. Walker, S.S. Bedi, S.K. Shah, F. Jimenez, H. Xue, J.A. Hamilton, P. Smith, C.P. Thomas, R.W. Mays, S. Pati, C.S. Cox, J. Neuroinflammation 9: 228 (2012).

[59] S. Pati, A.Y. Khakoo, J. Zhao, F. Jimenez, M.H. Gerber, M. Harting, J.B. Redell, R. Grill, Y. Matsuo, S. Guha, C.S. Cox Jr., M.S. Reitz Jr., J.B. Holcomb, P.K. Dash, Stem Cells Dev. 20(1) (2011) 89-101.

[60] T. Menge, Y. Zhao, J. Zhao, K. Wataha, M. Gerber, J. Zhang, P. Letourneau, J. Redell, L. Shen, J. Wang, Z. Peng, H. Xue, R. Kozar, C.S. Cox Jr., A.Y. Khakoo, J.B. Holcomb, P.K. Dash, S. Pati, Sci. Transl. Med. 4(161) (2012) 161ra150.

[61] P.A. Walker, S.K. Shah, F. Jimenez, M.H. Gerber, H. Xue, R. Cutrone, J.A. Hamilton, R.W. Mays, R. Deans, S. Pati, P.K. Dash, C.S. Cox Jr., Exp. Neurol. 225(2) (2010) 341-52. 